\title{
Suppression of vascular endothelial growth factor expression by cannabinoids in a canine osteosarcoma cell line
}

This article was published in the following Dove Press journal:

Veterinary Medicine: Research and Reports

4 July 2013

Number of times this article has been viewed

\author{
Andreza S Figueiredo' \\ Hiram J García-Crescioni' \\ Sandra C Bulla' \\ Matthew K Ross ${ }^{2}$ \\ Chelsea Mclntosh' \\ Kari Lunsford ${ }^{3}$ \\ Camilo Bulla' \\ 'Department of Pathobiology and \\ Population Medicine, ${ }^{2}$ Department \\ of Basic Sciences, ${ }^{3}$ Department \\ of Clinical Sciences and Animal \\ Health Center, College of Veterinary \\ Medicine, Mississippi State University, \\ Mississippi State, MS, USA
}

\begin{abstract}
Vascular endothelial growth factor (VEGF) is a key regulator in both physiologic and pathologic angiogenesis, and cannabinoids decrease VEGF release in human and murine cancer cells. The aim of this study was to assess the in vitro effects of a synthetic cannabinoid, WIN-55,212-2, on the expression of the proangiogenic factor VEGF-A in the canine osteosarcoma cell line 8 . After analysis of gene expression by quantitative real-time polymerase chain reaction, the compound decreased VEGF-A expression by $35 \% \pm 10 \%(P<0.0001)$ as compared with the control. This synthetic cannabinoid shows promise as a potential inhibitor of angiogenesis, and further studies are warranted to investigate its in vivo effects and to explore the potential of this and related compounds as adjuvant cancer therapy in the dog.
\end{abstract}

Keywords: dog, cancer, angiogenesis, cannabinoids

\section{Introduction}

Vascular endothelial growth factor (VEGF) is a proangiogenic signaling protein that induces proliferation and migration of vascular endothelial cells. The VEGF family comprises several glycoproteins, including VEGF-A, which is a major regulator of normal and abnormal angiogenesis. ${ }^{1}$ It plays a key role in tumor angiogenesis and has been found to be overexpressed in malignant tumors. ${ }^{2}$ Neoplastic tumors are dependent on angiogenesis because the formation of new blood vessels is required for delivery of the necessary nutrients and oxygen. In the absence of angiogenesis, tumors cannot grow larger than approximately $2 \mathrm{~mm}$ in diameter. Cells in rapidly growing masses are triggered by hypoxia to increase cellular VEGF expression in order to induce new vessel growth. ${ }^{3}$

Endocannabinoids, phytocannabinoids, and synthetic cannabinoids bind to cannabinoid $1\left(\mathrm{CB}_{1}\right)$ and $2\left(\mathrm{CB}_{2}\right)$ receptors, ${ }^{4}$ and can inhibit tumor growth in several cancers, including mouse glioma, ${ }^{5}$ and human pancreatic, ${ }^{6}$ the uterine, ${ }^{7}$ and prostatic cancer. ${ }^{8} \mathrm{CB}_{1}$ and $\mathrm{CB}_{2}$ receptors are found in many tissues in mammals, ${ }^{4}$ including the bone cells of human ${ }^{9}$ and mouse ${ }^{10}$ species. WIN-55,212-2 is a synthetic cannabinoid that binds to both $\mathrm{CB}_{1}$ and $\mathrm{CB}_{2}$ receptors and had been demonstrated to impede angiogenesis, induce apoptosis in mouse cancer cells, ${ }^{9}$ and inhibit VEGF expression in both human and mouse cancer cells. ${ }^{11,12}$

Cancer biology and tumor behavior have been shown to be similar in humans and dogs for several common cancers, ${ }^{13}$ and the dog is emerging as a valuable naturally occurring cancer model. ${ }^{14}$ Examples of these similarities are the canine and human leukemias and lymphomas, that have a similar clinical presentation, tumor biology, and response to therapy, and have been demonstrated to share evolutionary conserved 
chromosomal aberrations and conserved mutations within key oncogenes. ${ }^{15}$ Mammary malignancies share the same pattern of deregulated genes. ${ }^{16}$ Specifically, studies conducted with canine osteosarcoma were the first to yield results applicable to humans. ${ }^{17}$ The similarities between osteosarcoma in both species include a large patient size, $75 \%$ or more cases affecting the appendicular skeleton, metaphyseal location, less than $10 \%$ of patients having documented metastasis at presentation, over $90 \%$ of tumors showing high-grade histology, $75 \%$ of tumors showing aneuploidy, the metastatic rate being $80 \%$ or more with amputation alone, the lung being the most common site of metastasis, and improved survival with adjuvant chemotherapy, ${ }^{18}$ as well as deregulation of key cellular proteins, like STAT3. ${ }^{19}$ Recently, the study of molecular profiles derived from canine osteosarcoma helped to group complex human osteosarcoma into biologically and clinically relevant molecular subtypes. ${ }^{20}$

Despite aggressive treatment including surgery and chemotherapy, little improvement in survival time has been achieved in either humans or dogs. ${ }^{21,22}$ Added to the great importance of osteosarcoma in both humans and dogs described above is the fact that VEGF levels are predictive of pulmonary metastasis and a poor prognosis in human osteosarcoma, ${ }^{23}$ leading to the hypothesis that impairment of the proangiogenic pathway with cannabinoids can improve the prognosis of the disease.

To the best of our knowledge, there are no published studies evaluating VEGF levels in canine osteosarcoma or the effects of cannabinoids in canine cancer or on canine tumor cells. Thus, the aim of this study was to assess the impact of treatment with WIN-55,212-2 on VEGF-A expression in the canine osteosarcoma 8 (OSA-8) cell line. ${ }^{20}$ To the authors' knowledge, this is the first study using a canine model to evaluate the possible use of cannabinoids to impair angiogenesis in osteosarcoma.

\section{Materials and methods}

The OSA-8 cells were maintained in Dulbecco's Modified Eagle's Medium (Gibco, Grand Island, NY, USA) supplemented with 10\% fetal bovine serum (Gibco), 0.2\% Primocin (InvivoGen, San Diego, CA, USA), and 1\% HEPES (BioReagent, Sigma-Aldrich, St Louis, MO, USA). Twenty-four hours before the experiments, $8 \times 10^{6}$ cells were seeded per well. A stock solution of WIN-55,212-2 (Cayman Chemical Company, Ann Arbor, MI, USA) was prepared in dimethylsulfoxide (Thermo Fisher Scientific Inc, Waltham, MA, USA). The solution was added to OSA-8 cells to a final concentration of $1 \mu \mathrm{M}$ in supplemented Dulbecco's Modified Eagle's Medium and incubated for 48 hours $(n=8)$. Control incubations $(n=6)$ had the same dimethylsulfoxide content of $0.1 \%$ (v/v). Three independent experiments were performed.

After total RNA extraction (RNeasy Mini Kit, Qiagen, Valencia, CA, USA), RNA quality and concentration was evaluated by spectrophotometry. Real-time quantitative polymerase chain reaction was performed by a one-step reaction with a primer pair and labeled probe specific for canine VEGF-A (TaqMan Gene Expression Assay, Invitrogen, Carlsbad, CA, USA).

A specific canine GAPDH primer pair and labeled probe (Eurofins MWG Operon, Huntsville, AL, USA) was designed for normalization purposes. It was demonstrated to be a suitable gene for normalization under the experimental conditions after following procedures described elsewhere. ${ }^{24}$ GAPDH was considered to be stably expressed during the experiment on the basis of a-1.068-fold change because of treatment and checked by the Student's $t$-test with a $P$ value of 0.48 .

The comparative quantification method was used for relative quantification ${ }^{25}$ and all samples were run in triplicate. The amplification efficiencies were analyzed by 10 -fold dilution of a series of pooled RNA samples by $96.3 \%$ and $99.9 \%$ for VEGF-A and GAPDH, respectively.

Data are presented as the mean \pm standard deviation. The statistical analysis was done by analysis of variance followed by the Student's $t$-test ${ }^{26}$ using SAS version 9.2 (SAS Institute Inc, Cary, NC, USA). A value of $P<0.05$ was considered to be statistically significant.

\section{Results}

As shown in Figure 1, OSA-8 cells incubated with WIN-55,212-2 for 48 hours showed a decrease in VEGF-A expression by $35 \% \pm 10 \%$ on average $(P<0.0001)$ compared with the control (dimethyl sulfoxide), which was $100 \% \pm 23 \%$.

\section{Discussion}

In this paper, we detail the use of a highly sensitive and specific quantitative real-time polymerase chain reaction method for initial assessment of VEGF-A mRNA levels in a canine tumor cell line following treatment with a cannabinoid. We have demonstrated that WIN-55,212-2 decreased VEGF-A expression in the cultured OSA- 8 cells by $35 \%$. This finding is in accordance with other reports of the antiangiogenic effect of WIN-55,212-2, with a greater than $25 \%$ decrease in VEGF release from human and rat glioma cells, ${ }^{12}$ a $40 \%$ decrease from human prostate cancer cells, ${ }^{8}$ and marked inhibition of VEGF expression in human skin carcinoma. ${ }^{11}$

In addition to the antiangiogenic effects of cannabinoids observed here and previously, these compounds have been 


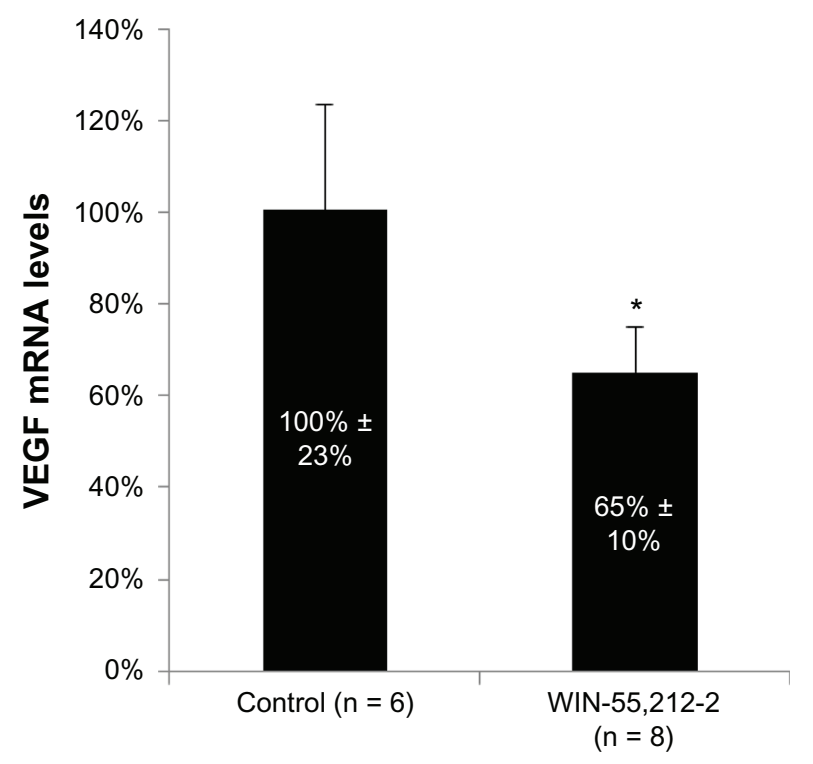

Figure I Comparison between vascular endothelial growth factor (VEGF) messenger RNA (mRNA) levels in control OSA-8 cells cultured for 48 hours with dimethylsulfoxide $(0.1 \%$, v/v) or WIN-55,2I2-2 (I $\mu \mathrm{M})$.

Notes: Data are reported as the mean \pm standard deviation of three independent experiments. *Statistically significant at $P<0.05$.

used successfully in patients with human cancer for the treatment of symptoms resulting from both cancer and antineoplastic therapies, including nausea and vomiting, weight loss, lack of appetite, and pain. Although these benefits have been known for years, it is only recently that the clinical anticancer effects of cannabinoids have been investigated. ${ }^{4}$

Our findings give rise to at least two further lines of research. First is the need to address the role of VEGF in the establishment of canine osteosarcoma and development of metastasis. Second is the need to elucidate the exact signaling mechanisms governing the inhibitory effects of cannabinoids on VEGF expression, although the involvement of cannabinoid receptors has been suggested by the fact that $\mathrm{CB}_{1}$ and $\mathrm{CB}_{2}$ antagonists block the beneficial action of WIN-55,212-2 in skin carcinoma. ${ }^{12}$ Further research is currently underway in our laboratory with WIN-55,212-2 and the OSA-8 cell line to assess its precise mechanism of action.

Canine osteosarcoma accounts for approximately $85 \%$ of all canine skeletal tumors and, although advances in disease management have been achieved, $40 \%-50 \%$ of dogs treated by amputation followed by adjuvant therapy survive about one year and only $10 \%-20 \%$ survive more than 2 years. ${ }^{27}$ In the United States, the number of new cases is estimated to exceed 10,000 per year, representing 10 times more than that in people. ${ }^{18,19}$ These, in addition to the finding that VEGF levels in human osteosarcoma are indicative of a poor prognosis ${ }^{22}$ and given the similarities between canine and human osteosarcoma, ${ }^{18}$ make the canine OSA-8 cell line an attractive in vitro model for assessing the antiangiogenic effects of cannabinoid treatment, and future work will benefit both comparative angiogenesis research and canine patients with cancer.

\section{Conclusion}

In conclusion, the cannabinoid agonist, WIN-55,212-2, was shown to reduce the expression of VEGF-A in a canine osteosarcoma cell line. On the basis of the results reported here, we suggest that cannabinoid receptor agonists may have the potential for use as clinical angiogenesis inhibitors. Our results suggest a first step toward the use of cannabinoids as potential adjuvants to chemotherapeutics in the treatment of canine cancers.

\section{Acknowledgment}

We thank Jaime F Modiano for kindly providing the canine osteosarcoma cell line. This study was supported by the Merial Veterinary Scholars Program and the Mississippi State University, College of Veterinary Medicine.

\section{Disclosure}

The authors report no conflicts of interest in this work.

\section{References}

1. Ferrara N, Kerbel RS. Angiogenesis as a therapeutic target. Nature. 2005;438:967-974.

2. Ferrara N, Davis-Smyth T. The biology of vascular endothelial growth factor. Endocr Rev. 1997;18:4-25.

3. Battinelli EM, Markens BA, Italiano JE Jr. Release of angiogenesis regulatory proteins from platelet alpha granules: modulation of physiologic and pathologic angiogenesis. Blood. 2011;118: 1359-1369.

4. Velasco G, Sánchez C, Guzmán M. Towards the use of cannabinoids as antitumor agents. Nat Rev Cancer. 2012;12:436-444.

5. Galve-Roperh I, Sanchez C, Cortez ML, et al. Antitumoral action of cannabinoids: involvement of sustained ceramide accumulation and extracellular signal-regulated kinase activation. Nat Med. 2000;6: 313-319.

6. Carracedo A, Gironella M, Lorente M, et al. Cannabinoids induce apoptosis of pancreatic tumor cells via endoplasmic reticulum stress-related genes. Cancer Res. 2006;66:6748-6755.

7. Cianchi R, Papucci L, Schiavone N, et al. Cannabinoid receptor activation induces apoptosis through tumor necrosis factor alpha-mediated ceramide de novo synthesis in colon cancer cells. Clin Cancer Res. 2008;14: 7691-7700.

8. Sarfaraz S, Afaq F, Adhami VM, et al. Cannabinoid receptor as a novel target for the treatment of prostate cancer. Cancer Res. 2005;65: 1635-1641.

9. Whyte LS, Ford L, Ridge SA, et al. Cannabinoid and bone: endocannabinoid modulate human osteoclast function in vitro. Br J Pharmacol. 2012; 165:2584-2597.

10. Idris AI, Sophocleous A, Landao-Bassonga E, et al. Cannabinoid receptor type 1 protects against age-related osteoporosis by regulating osteoblast and adipocyte differentiation in marrow stromal cells. Cell Metab. 2009;10:139-147. 
11. Casanova ML, Blázquez C, Matínez-Palacio, et al. Inhibition of skin tumor growth and angiogenesis in vivo by activation of cannabinoid receptors. J Clin Invest. 2003;111:43-50.

12. Blázquez C, González-Feria L, Álvarez L, et al. Cannabinoids inhibit the vascular endothelial growth factor pathway in gliomas. Cancer Res. 2004;64:5617-5623.

13. Paoloni M, Khanna C. Translation of new cancer treatments from pet dogs to humans. Nat Rev Cancer. 2008;8:147-156.

14. Gordon I, Paoloni M, Mazcko C, Khanna C. The Comparative Oncology Trials Consortium: using spontaneously occurring cancers in dogs to inform the cancer drug development pathway. PLoS Med. 2009;6:e1000161.

15. Modiano JF, Breen M. Shared pathogenesis of human and canine tumors - an inextricable link between cancer and evolution. Cancer Ther. 2008;6:239-246.

16. Uva P, Aurisicchio L, Watters J, et al. Comparative expression pathway analysis of human and canine mammary tumors. BMC Genomics. 2009;10:135-155.

17. LaRue SM, Withrow SJ, Powers BE, et al. Limb-sparing treatment for osteosarcoma in dogs. J Am Vet Med Assoc. 1989;195:1734-1744.

18. Withrow SJ, Powers BE, Straw RC, Wilkins RM. Comparative aspects of osteosarcoma. Dog versus man. Clin Orthop Relat Res. 1991;270: 159-168.

19. Fossey SL, Liao AT, McCleese JK, et al. Characterization of STAT3 activation and expression in canine and human osteosarcoma. $B M C$ Cancer. 2009;9:81-95.
20. Scott MC, Sarver AL, Gavin KJ, et al. Molecular subtypes of osteosarcoma identified by reducing tumor heterogeneity through an interspecies comparative approach. Bone. 2011;49:366-367.

21. Clark JC, Dass CR, Choong PF. A review of clinical and molecular prognostic factors in osteosarcoma. J Cancer Res Clin Oncol. 2008;134: 281-297.

22. Chun R, Lorimier LP. Update on the biology and management of canine osteosarcoma. Vet Clin North Am Small Anim Pract. 2003;33: 491-516.

23. Kaya M, Wada T, Akatsuka T, et al. Vascular endothelial growth factor expression in untreated osteosarcoma is predictive of pulmonary metastasis and poor prognosis. Clin Cancer Res. 2000;6:572-577.

24. Schmittgen TD, Livak KJ. Analyzing real-time PCR data by the comparative CT method Thomas. Nat Protoc. 2008;3:1101-1108.

25. Livak KJ, Schmittgen TD. Analysis of relative gene expression data using real-time quantitative PCR and the 2- $\Delta \Delta \mathrm{Ct}$ method. Methods. 2001;25:402-408.

26. Yuan JS, Reed A, Chen F, Stewart N Jr. Statistical analysis of real-time PCR data. BMC Bioinformatics. 2006;7:85-96.

27. Phillips B, Powers BE, Dernell WS, et al. Use of single-agent carboplatin as adjuvant or neoadjuvant therapy in conjunction with amputation for appendicular osteosarcoma in dogs. J Am Anim Hosp Assoc. 2009;45: 33-38.
Veterinary Medicine: Research and Reports

\section{Publish your work in this journal}

Veterinary Medicine: Research and Reports is an international, peer-reviewed, open access journal publishing original research, case reports, editorials, reviews and commentaries on all areas of veterinary medicine. The manuscript management system is completely online and includes a very quick and fair peer-review system.

\section{Dovepress}

Visit http://www.dovepress.com/testimonials.php to read real quotes from published authors. 